\title{
CrystEngComm
}

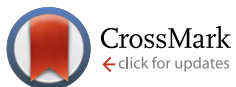

Cite this: CrystEngComm, 2015, 17, 3772

Received 12th March 2015,

Accepted 15th April 2015

DOI: 10.1039/c5ce00519a

\section{A simple strategy for the synthesis of well-defined bassanite nanorods $\uparrow$}

\author{
U. Tritschler, ${ }^{a}$ M. Kellermeier, ${ }^{\star b}$ C. Debus, ${ }^{a}$ A. Kempter ${ }^{c}$ and H. Cölfen ${ }^{\star a}$
}

www.rsc.org/crystengcomm

Bassanite is a metastable but industrially important form of calcium sulfate, which is commonly produced by heating of gypsum. Here we show that pure bassanite can also be obtained at ambient conditions by quenching aqueous $\mathrm{CaSO}_{4}$ solutions in ethanol. This highlights that organic solvents can actually induce the formation of metastable phases rather than freezing precipitation processes.

Calcium sulfate occurs in considerable amounts as geological deposits in the form of gypsum $\left(\mathrm{CaSO}_{4} \cdot 2 \mathrm{H}_{2} \mathrm{O}\right)$ and anhydrite (anhydrous $\mathrm{CaSO}_{4}$ ). ${ }^{1}$ Both of these minerals are used for the large-scale production of plasters, fertilisers, and cements. ${ }^{2}$ The third crystalline polymorph of calcium sulfate, the hemihydrate $\left(\mathrm{CaSO}_{4} \cdot 0.5 \mathrm{H}_{2} \mathrm{O}\right.$, also known as bassanite), is much less abundant in Nature, but still represents one of the most extensively produced inorganic materials worldwide, mainly due to its broad application for construction purposes. ${ }^{3}$ Usually, bassanite is obtained by heating of gypsum at temperatures between 100 and $150{ }^{\circ} \mathrm{C}$ in dry state ${ }^{4-6}$ or at $75{ }^{\circ} \mathrm{C}$ in methanol-water solutions, ${ }^{7}$ leading to partial release of hydration water. Upon subsequent addition of water, it transforms back into gypsum in a rapid exothermic hydration process, $^{2,8,9}$ which accounts for the setting and hardening of plaster and cement materials.

The crystallisation of calcium sulfate from aqueous solutions has been studied in detail over the past decades, mostly assuming classical concepts of nucleation and growth, i.e. direct formation of the thermodynamically stable phase (which is gypsum under ambient conditions). ${ }^{2,10-12}$ In recent years, there is increasing evidence that mineralisation can also proceed through alternative reaction channels, which

\footnotetext{
${ }^{a}$ University of Konstanz, Physical Chemistry, Universitätsstraße 10, D-78457 Konstanz, Germany. E-mail: helmut.coelfen@uni-konstanz.de ${ }^{b}$ Material Physics, BASF SE, GMC/O - G201, 67056 Ludwigshafen, Germany. E-mail: matthias.kellermeier@basf.com

${ }^{c}$ Reactive Systems and Inorganic Nanomaterials Research, BASF SE, GMV/N - J550, 67056 Ludwigshafen, Germany

$\dagger$ Electronic supplementary information (ESI) available: Experimental details, figures and tables. See DOI: 10.1039/c5ce00519a
}

may involve metastable polymorphs as temporary intermediates and rely on nanoparticle- or cluster-based mechanisms. ${ }^{13,14}$ Indeed, related observations were made for the calcium sulfate system in a number of very recent studies, where multi-stage precipitation pathways including amorphous precursor phases ${ }^{15-19}$ and/or bassanite intermediates ${ }^{15,18-20}$ were identified. In the latter case, the transformation into stable gypsum was found to occur via oriented attachment and subsequent merging of bassanite nanoparticles into micron-sized gypsum needles, ${ }^{20,21}$ a process consistent with the notion of mesocrystals and general models of nonclassical crystallisation. ${ }^{13}$ We note, however, that these conclusions were drawn based on data obtained by a quenching process using organic solvents, which was supposed to arrest any ongoing processes during crystallisation. ${ }^{15,19,20}$ Inspired by these findings and in light of the industrial importance of bassanite, we have developed a new strategy to synthesise well-defined bassanite nanorods of uniform shape and size via a scalable solution-based process at room temperature. This was achieved by quenching supersaturated solutions of $\mathrm{CaSO}_{4}$ in an excess of ethanol. In addition to devising a promising alternative route for the production of pure bassanite, our results strongly suggest that quenching of crystallising aqueous solutions with solvents like ethanol is a highly critical step, as it may actually induce the formation and/or stabilisation of metastable phases (like bassanite in the present case), rather than simply freezing the situation in solution, as often assumed.

In a typical experiment, equal volumes $(25 \mathrm{~mL})$ of aqueous $\mathrm{CaCl}_{2}$ and $\mathrm{Na}_{2} \mathrm{SO}_{4}$ solutions (both $\left.50 \mathrm{mM}\right)^{22}$ were mixed and, after brief shaking, poured into $500 \mathrm{~mL}$ ethanol (see section $\mathrm{S} 1$ in the ESI $\dagger$ for experimental details). This led to an instant clouding of the initially translucent samples, indicating immediate formation of $\mathrm{CaSO}_{4}$ particles upon this drastic change in solvent composition. The dispersed particles were isolated by centrifugation and, after drying in vacuum, analysed by a number of different techniques. Corresponding results are compiled in Fig. 1. Infrared (IR) spectroscopy and 
$\mathrm{X}$-ray diffraction (XRD) patterns demonstrate that the formed material is virtually phase-pure bassanite, ${ }^{18}$ with no signs for traces of gypsum being discernible (Fig. 1a-b). Transmission (TEM) and scanning electron microscopy (SEM) images show a quite uniform population of rod-like nanoparticles with lengths between 50 and $200 \mathrm{~nm}$ and typical widths of 25-40 nm (Fig. 1c, e, f), which were further confirmed to be bassanite by means of electron diffraction (ED; Fig. 1d). A closer look at individual particles reveals a porous texture, with voids of 20-25 $\mathrm{nm}$ in diameter ( $c f$. inset in Fig. 1c and f). The size and morphology of these particles, as well as their porosity, are widely identical to what has been described for bassanite nanorods that were observed during $\mathrm{CaSO}_{4}$ crystallisation from purely aqueous solutions and claimed to be stable precursors of the final gypsum phase. ${ }^{15,18-20}$ Moreover, Yang et al. obtained similar nanoparticles when precipitating calcium sulfate from water/ethanol mixtures at elevated temperature, but could not demonstrate the formation of pure bassanite via this strategy. ${ }^{23}$

Overall, these experiments demonstrate that addition of $50 \mathrm{~mL} 25 \mathrm{mM} \mathrm{CaSO}_{4}$ solution to an initial volume of $500 \mathrm{~mL}$ ethanol results in the formation of $100 \%$ bassanite in fairly

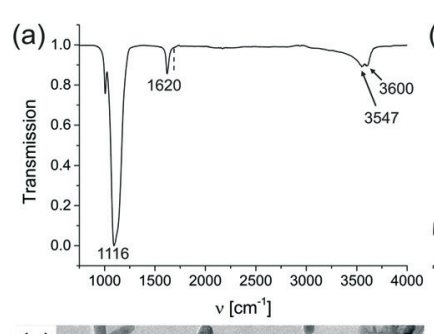

(b)
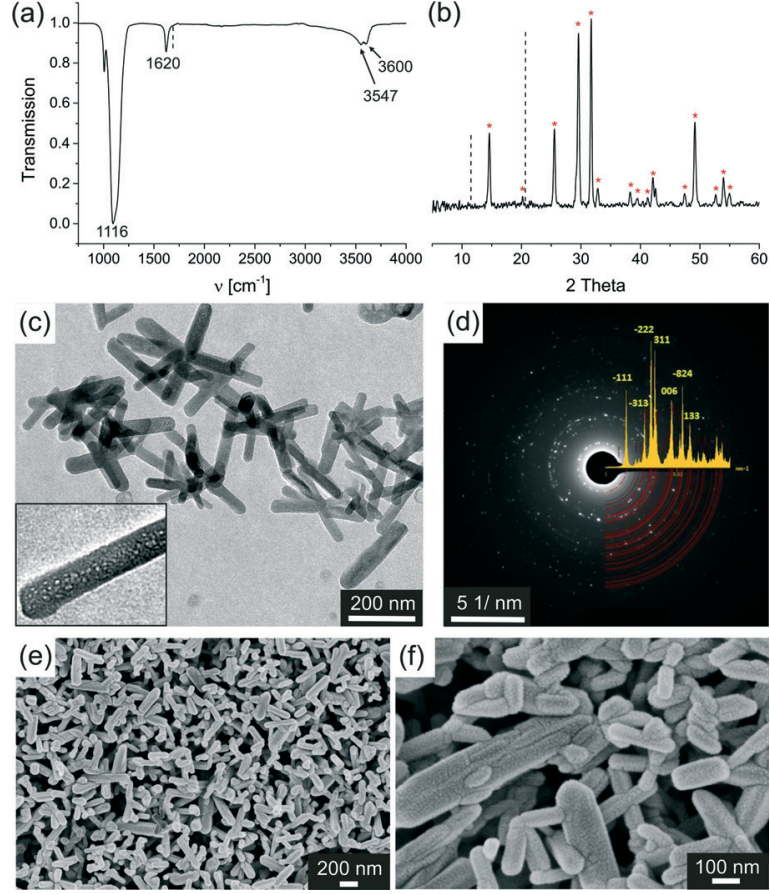

Fig. 1 Characterisation of solid products obtained by quenching $25 \mathrm{mM}$ CaSO4 solution into an excess of ethanol at final water contents of $16-27 \mathrm{wt} \%$. (a) IR spectrum of the precipitates, showing typical bands of bassanite (the dashed line marks the position of a characteristic peak appearing only in the presence of gypsum). (b) XRD pattern of the product, wherein reflections belonging to bassanite are highlighted with red asterisks and dashed lines indicate the most intense peaks expected for gypsum. (c) TEM image of the precipitates, revealing uniform rod-like nanoparticles that occasionally exhibit pronounced porosity (inset). (d) Corresponding electron diffraction pattern, displaying reflections that can all be indexed to bassanite. (e-f) SEM images of the bassanite particles at different magnifications. large amounts ( $c a .0 .2 \mathrm{~g}$ ), while no gypsum is obtained as a by-product. Therefore, this protocol was used as a starting point to study the reaction in more detail and further optimise the yield of bassanite in the syntheses. To that end, different experimental parameters were systematically varied, primarily the concentration of the added $\mathrm{CaSO}_{4}$ solution and the water content in the final solvent mixture. Results of these experiments are summarised in Fig. 2, which shows a $3 \mathrm{D}$ plot illustrating the amount of bassanite detected in the product ( $z$-axis, in wt\% relative to gypsum) as a function of the water content ( $x$-axis, in wt\% relative to ethanol in the final solvent mixture), and the concentration of the $\mathrm{CaSO}_{4}$ solution ( $y$-axis, in $\mathrm{mM}$ before quenching into ethanol). The yield of bassanite was determined from IR spectra of the obtained solids by using the intensity of the $v_{1}$ band at $1684 \mathrm{~cm}^{-1}$, which only occurs for gypsum. ${ }^{17,18}$ Intensity values were correlated with bassanite/gypsum mass ratios by measuring a series of defined mixtures of the two polymorphs. It was found that the intensity of the $v_{1}$ peak decreases exponentially with increasing bassanite content, so that a corresponding fit of the data could be used as calibration curve (see Fig. S1 in the ESI $\dagger$ ). The error associated with this procedure is estimated to $c a$. $\pm 10 \%$.

Essentially, the main product obtained for final water contents ranging from 16 to $33 \mathrm{wt} \%$ was bassanite, regardless of the $\mathrm{CaSO}_{4}$ concentration. In all these cases, the apparent yield was $>90 \%$, which corresponds to a virtually phase-pure material with regard to the limits of experimental error. When the water content was further increased to $41 \mathrm{wt} \%$, the bassanite fraction decreased to $c a$. $60 \%$ for $75 \mathrm{mM} \mathrm{CaSO}_{4(\mathrm{aq})}$, and to less than 20 and $10 \%$ for 50 and $25 \mathrm{mM}$, respectively. However, for $\mathrm{CaSO}_{4}$ concentrations of 100 and $150 \mathrm{mM}$, bassanite was still the main phase obtained, although certain amounts of gypsum crystals could be detected in these samples. Starting from $43 \mathrm{wt} \%$ water in the final dispersions, the bassanite yield was progressively lowered at all $\mathrm{CaSO}_{4}$ concentrations investigated, although the trend of higher

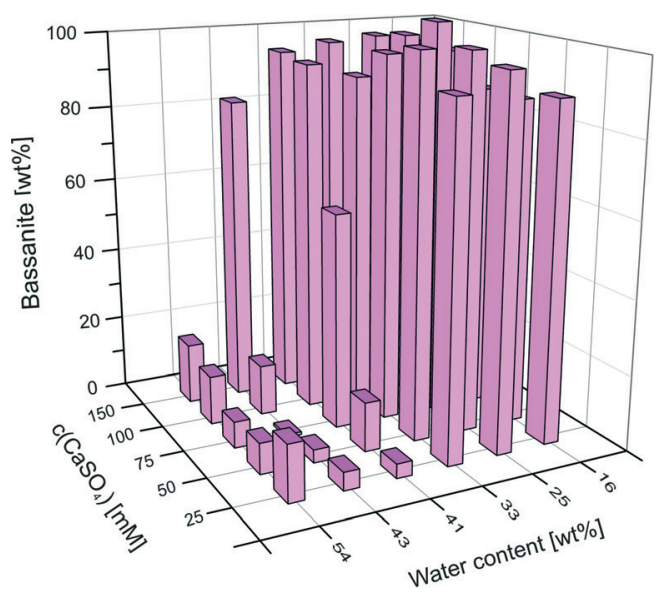

Fig. 2 Bassanite content of precipitates formed upon addition of aqueous calcium sulfate solutions (with variable concentrations) into ethanol/water mixtures at different final water contents. 
bassanite fractions in samples containing more $\mathrm{CaSO}_{4}$ is still apparent. At $54 \mathrm{wt} \%$ water, the measured bassanite/gypsum ratios were below $20 \%$ at all $\mathrm{CaSO}_{4}$ contents. Analyses of isolated solids confirmed that gypsum was the dominant polymorph in these samples, while bassanite was hardly detectable in most cases (see Fig. S2 in the ESI $\dagger$ ). Taken together, these data show that quenching of aqueous calcium sulfate solutions into ethanol yields pure bassanite as long as the total water content in the final dispersions does not exceed a certain limit. According to the present results, this threshold lies between 33 and $41 \mathrm{wt} \%$. This suggests that ethanol plays a crucial role in stabilising formed bassanite nanoparticles. On the other hand, as the initial aqueous $\mathrm{CaSO}_{4}$ solutions were all clear and transparent with no sign of turbidity, ethanol obviously induces the precipitation of bassanite from solution, ${ }^{24}$ while subsequently protecting it against transformation to gypsum. In any case, from a technical point of view, our data indicate that syntheses of bassanite via the developed protocol should be conducted at $150 \mathrm{mM} \mathrm{CaSO}_{4(\mathrm{aq})}$ and $41 \mathrm{wt} \%$ water, as this requires the lowest amount of organic solvent and affords the highest possible absolute yield of pure bassanite.

Replacing ethanol as the organic solvent by methanol, isopropanol, or acetone did not affect the yield of bassanite at a final water content of $16 \mathrm{wt} \%$, as confirmed by IR analyses (see Fig. S3 in the ESI $\dagger$ ). By contrast, when tetrahydrofuran (THF) was used as solvent, the obtained solids contained about $67 \%$ gypsum. This means either that THF is less capable of inhibiting the bassanite-to-gypsum transformation, or that it favours the kinetic formation of bassanite over gypsum to a lower extent than the other solvents studied. At $33 \mathrm{wt} \%$ water, still $100 \%$ bassanite was obtained with methanol (as for ethanol, $c f$. Fig. 2). In the case of isopropanol and acetone, however, the yield of bassanite was markedly reduced under these conditions, leading to measured bassanite fractions of 55\% (isopropanol) and 75\% (acetone). Interestingly, the observed trend in efficiency of the different solvents to generate and/or stabilise bassanite directly correlates with their polarity, as demonstrated by the following order of the solvents according to their suitability for bassanite synthesis: methanol $(\varepsilon=33.0) \approx$ ethanol $(\varepsilon=25.3)>$ acetone $(\varepsilon=21.0)>$ isopropanol $(\varepsilon=20.8)>\mathrm{THF}(\varepsilon=7.5)$, where $\varepsilon$ is the dielectric constant of the solvent ${ }^{25}$ at $25{ }^{\circ} \mathrm{C}(\varepsilon=80.1$ for water). Fig. 3 shows a plot of the bassanite yield as a function of the solvent polarity.

In other words, the better the miscibility of the solvent with water (or respectively the higher its mixing enthalpy with water), the more effective will the solvent be in terms of bassanite formation. This behaviour can be understood when considering that bassanite contains less hydration water than gypsum and, hence, that more water has to be removed from any solution (or amorphous solid) precursors to form bassanite. Consequently, solvents that are more prone to withdraw water (i.e. more polar ones) should be more suitable for the preparation of bassanite via the applied methodology, in line with what is observed experimentally. Such a

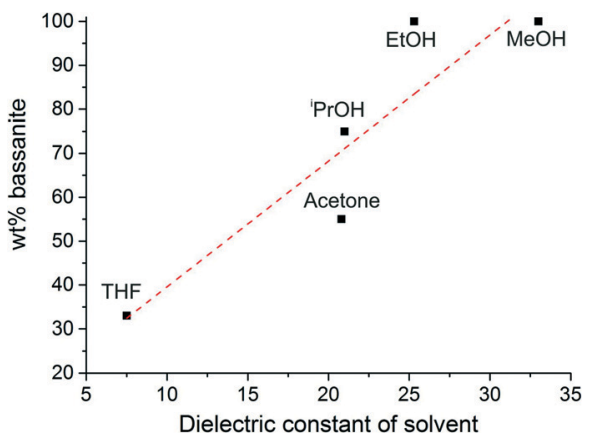

Fig. 3 Correlation between the bassanite fraction in the product and the dielectric constant of the used organic solvent. Note that the plot was constructed with data from batches with different water contents (cf. Fig. S3 in the $\mathrm{ESI} \dagger$ ).

dehydration-based scenario could furthermore explain the porosity of the bassanite nanoparticles, as the voids in the rods may result from rapid removal of water from calcium sulfate species precipitating under kinetically controlled, non-equilibrium conditions. In this regard, it seems as if the primary role of the organic solvents is to favour the crystallisation of bassanite over that of gypsum. The fact that this effect already occurs at water contents far beyond the stoichiometry of the minerals $\left(30-40 \mathrm{wt} \% \mathrm{H}_{2} \mathrm{O}\right.$ at $\leq 0.02 \mathrm{wt} \%$ $\mathrm{CaSO}_{4}$ ) underlines the importance of kinetics in these systems and suggests that nucleation and/or growth of gypsum is actively inhibited in the alcohol-containing media (as in principle more than enough water would be present for gypsum, the actually stable phase, to be formed).

Interestingly, early studies on the precipitation of $\mathrm{CaSO}_{4}$ in pure alcohol $(\mathrm{MeOH}$ in this case) reported the formation of solvate adducts with a crystal structure akin to that of bassanite, but containing methanol molecules instead of water in the lattice. ${ }^{26}$ This, however, was not observed in the present work even at low final water contents, again suggesting that dehydration of water-rich solute (or solid) $\mathrm{CaSO}_{4}$ precursors is a key step in the formation of bassanite in excess organic solvent.

Given that bassanite is metastable with respect to gypsum, ${ }^{2}$ we have tested the temporal stability of the bassanite nanoparticles obtained at low water contents. In dry state, there was no noticeable transformation into gypsum over a period of 3 weeks (see Fig. S4 in the ESI $\dagger$ ). In dispersion, the bassanite particles proved to be stable for about 4 weeks at $50 \mathrm{mM} \mathrm{CaSO}_{4(\mathrm{aq})}$ and $16 \mathrm{wt} \% \mathrm{H}_{2} \mathrm{O}$ (see Fig. S5a in the ESI $\dagger$ ) in the absence of any stabilising additives and despite continuous stirring. By contrast, progressive transformation of bassanite into gypsum could be observed when the $\mathrm{CaSO}_{4}$ concentration was increased or longer ageing times were chosen (see Fig. S5b in the ESI $\dagger$ ). In order to study the mechanism of bassanite-to-gypsum transformation in more detail, the synthesis parameters were tuned such that conversion to gypsum occurred within a reasonable frame of time, allowing for convenient sampling of the process. This was found to be possible at a $\mathrm{CaSO}_{4}$ concentration of $50 \mathrm{mM}$ and a final water 

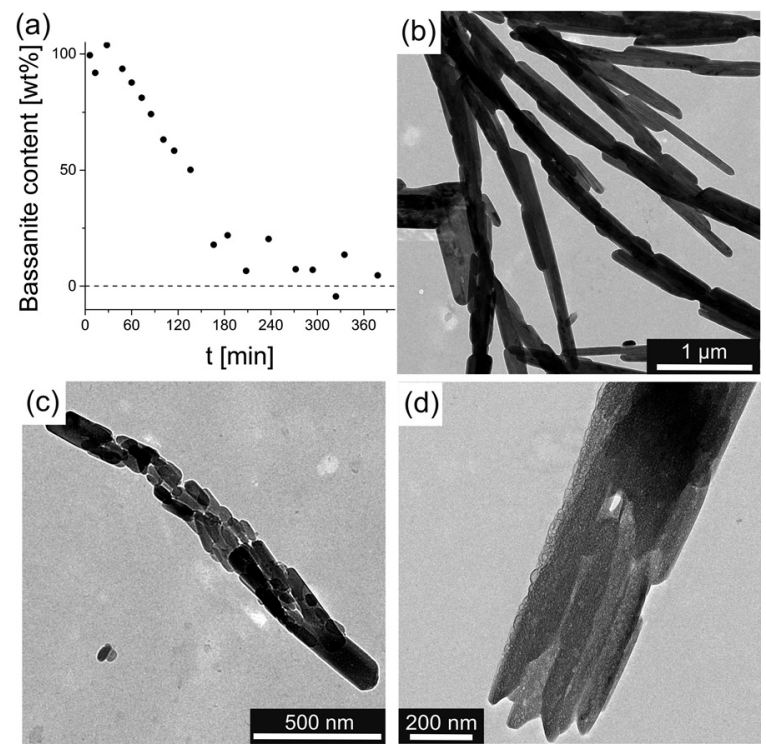

Fig. 4 Transformation of bassanite nanoparticles into micron-sized gypsum crystals. (a) Fraction of bassanite detected by IR as a function of ageing time in an ethanolic dispersion with a water content of $33 \mathrm{wt} \%$. (b-d) TEM images showing the supposedly crucial step in the transformation process, i.e. oriented attachment of bassanite nanorods leading to crystallographically aligned particle assemblies that later merge to yield crystalline gypsum.

content of $33 \mathrm{wt} \%$. Here, it took approximately 3 hours for transformation to be completed, as indicated by timedependent bassanite fraction, which show a roughly linear decrease from 100 to $\sim 0 \mathrm{wt} \%$ (Fig. 4a). On the other hand, TEM micrographs of samples collected during the transformation process clearly show that the initially present bassanite nanorods convert into gypsum via an aggregationbased process, in which individual particles first assemble and align parallel to the long axes of the rods (Fig. $4 \mathrm{~b}-\mathrm{d}$ ), to subsequently merge in a common crystallographic register and collectively transform into gypsum. In this regard, our data directly confirm the notion that gypsum crystals are formed from bassanite nanorods via particle aggregation and oriented attachment, as observed in previous studies. ${ }^{20} \mathrm{How}^{-}$ ever, while this mechanism appears to be the preferential reaction pathway for the formation of gypsum starting from an existing population of bassanite nanoparticles, it does not necessarily apply for gypsum crystallisation from purely aqueous media in general. Our experiments provide clear evidence that the formation of bassanite is induced, or at least favoured, by organic solvents. In fact, the same solvents (especially ethanol) have been used in previous studies to supposedly "quench" calcium sulfate precipitation in aqueous systems, ${ }^{15,18-20}$ however without explicitly assessing direct effects of the solvents on polymorph selection and other structural or morphological properties of the resulting particles. In light of the present work, data obtained by such quenching techniques should be considered with care in order to exclude possible preparation artefacts.

In summary, we have introduced a new method for the synthesis of phase-pure bassanite via a straightforward solution-based process at room temperature. Specifically, the rapid change in solvent polarity caused by pouring aqueous $\mathrm{CaSO}_{4}$ solutions into an excess of ethanol was found to produce crystalline bassanite nanorods with quite uniform sizes. This approach might serve as a promising green alternative to the commonly applied process of heating gypsum, which requires considerable energy input and moreover affords a rather ill-defined micron-sized material (see Fig. S6 in the ESI $\dagger$ ). The bassanite nanoparticles prepared by the new procedure are expected to exhibit higher effective surface areas and thus take up water more efficiently during hydration. This should lead to quicker hardening of corresponding plasters, and might be useful for certain construction applications. Moreover, the results of the present study have clearly shown that organic solvents can have substantial impact on $\mathrm{CaSO}_{4}$ polymorph selection and affect precipitation kinetics. This is an important finding as it suggests that the use of these solvents in the isolation of transient crystallisation intermediates can in fact cause the formation of these presumed intermediates, rather than freezing the actual processes occurring in aqueous solution, as desired. This is of course critical to data interpretation and highlights the drawbacks of quenching techniques in the study of crystallisation mechanisms, be it for calcium sulfate or any other mineral system.

\section{Acknowledgements}

The authors thank Elena Rosseeva for help with electron diffraction analyses and Maria Helminger, Igor Zlotnikov and Masoud Farhadi Khouzani for XRD measurements. We are grateful to Alexander van Driessche, Juan Ma García-Ruiz, and Liane Benning for valuable discussions. Parts of this work were funded by the DFG (Project SPP1420) and BASF SE.

\section{Notes and references}

1 A. E. S. Van Driessche, J. M. Garcia-Ruiz, K. Tsukamoto, L. D. Patino-Lopez and H. Satoh, Proc. Natl. Acad. Sci. U. S. A., 2011, 108, 15721-15726.

2 D. Freyer and W. Voigt, Monatsh. Chem., 2003, 134, 693-719.

3 H. Weiss and M. F. Bräu, Angew. Chem., Int. Ed., 2009, 48, 3520-3524.

4 E. Badens, P. Llewellyn, J. M. Fulconis, C. Jourdan, S. Veesler, R. Boistelle and F. Rouquerol, J. Solid State Chem., 1998, 139, 37-44.

5 M. Isida, M. Kamata and T. Shirai, J. Chem. Eng. Jpn., 1970, 3, 201-206.

6 J. Bensted and S. Prakash, Nature, 1968, 219, 60-61.

7 B. Guan, G. Jiang, Z. Wu, J. Mao and B. Kong, J. Am. Ceram. Soc., 2011, 94, 3261-3266.

8 T. Nissinen, M. Li, N. Brielles and S. Mann, CrystEngComm, 2013, 15, 3793-3798.

9 L. Amathieu and R. Boistelle, J. Cryst. Growth, 1988, 88, 183-192.

10 S.-T. Liu and G. H. Nancollas, J. Cryst. Growth, 1970, 6, 281-289. 
11 A. Packter, J. Cryst. Growth, 1974, 21, 191-194.

12 P. G. Klepetsanis and P. G. Koutsoukos, J. Cryst. Growth, 1989, 98, 480-486.

13 H. Cölfen and M. Antonietti, Mesocrystals and Nonclassical Crystallization, John Wiley \& Sons, Ltd, New York, 2008.

14 J. Baumgartner, A. Dey, P. H. H. Bomans, C. Le Coadou, P. Fratzl, N. A. J. M. Sommerdijk and D. Faivre, Nat. Mater., 2013, 12, 310-314.

15 Y.-W. Wang, Y.-Y. Kim, H. K. Christenson and F. C. Meldrum, Chem. Commun., 2012, 48, 504-506.

16 A. Saha, J. Lee, S. M. Pancera, M. F. Bräu, A. Kempter, A. Tripathi and A. Bose, Langmuir, 2012, 28, 11182-11187.

17 F. Jones, CrystEngComm, 2012, 14, 8374-8381.

18 Y.-W. Wang and F. C. Meldrum, J. Mater. Chem., 2012, 22, 22055-22062.

19 Y.-W. Wang, H. K. Christenson and F. C. Meldrum, Adv. Funct. Mater., 2013, 23, 5615-5623.

20 A. E. S. Van Driessche, L. G. Benning, J. D. RodriguezBlanco, M. Ossorio, P. Bots and J. M. García-Ruiz, Science, 2012, 336, 69-72.
21 H. Zhang and J. F. Banfield, J. Phys. Chem. Lett., 2012, 3, 2882-2886.

22 All aqueous $\mathrm{CaCl}_{2} / \mathrm{Na}_{2} \mathrm{SO}_{4}$ precursor solutions were supersaturated with respect to gypsum, while the saturation limit of bulk bassanite was only exceeded at the two highest $\mathrm{CaSO}_{4}$ concentrations investigated (see Table S1 in the ESI $\dagger$ ). Nonetheless, all samples were clear and transparent metastable solutions with no visual indication of precipitation, i.e. nucleation had apparently not yet taken place when the aqueous mixtures were quenched into ethanol.

23 L.-X. Yang, Y.-F. Meng, P. Yin, Y.-X. Yang, Y.-Y. Tang and L.-F. Qin, Bull. Mater. Sci., 2011, 34, 233-237.

24 Note that fairly large amounts of bassanite were obtained after quenching, whereas the number of particles in solution before quenching was too low to be detected by scattering techniques.

25 D. R. Lide, CRC Handbook of Chemistry and Physics, CRC Press, 84 edn, 2003-2004.

26 K. Reisdorf and W. Abriel, ZKG Int., 1988, 41, 356-357. 